\title{
Trading off health and financial protection benefits with multiobjective optimization
}

\author{
Özlem Karsu' ${ }^{1}$ @ | Alec Morton² ${ }^{2}$
}

${ }^{1}$ Department of Industrial Engineering, Bilkent University, Ankara, Turkey

${ }^{2}$ Management Science Department, University of Strathclyde Business School, Glasgow, UK

\section{Correspondence}

Özlem Karsu, Department of Industrial Engineering, Bilkent University, Ankara, Turkey.

Email: ozlemkarsu@bilkent.edu.tr

\begin{abstract}
Countries which are introducing a system of Universal health coverage have to make a number of key tradeoffs, of which one is the tradeoff between the level of coverage and the degree to which patients are exposed to potentially catastrophic financial risk. In this study, we first present a way in which decision makers might be supported to focus on in a particular part of the tradeoff curve and ultimately choose an efficient solution. We then introduce some multiobjective optimization models for generating the tradeoff curves given data about potential treatment numbers, costs, and benefits. Using a dataset from Malawi, we demonstrate the approach and suggest a core index metric to make specific observations on the individual treatments. Moreover, as there has been some debate about the best way to measure financial exposure, we also investigate the extent to sensitivity of our results to the precise technical choice of financial exposure metric. Specifically, we consider two metrics, which are the total number of cases protected from catastrophic expenditure and a convex penalty function that penalizes out-of-pocket expenditures in an increasingly growing way, respectively.
\end{abstract}

\section{K E Y W O R D S}

cost-effectiveness analysis, economic analysis, extended cost-effectiveness analysis, financial protection, health benefits packages

\section{1 | INTRODUCTION}

The ideal healthcare system would pay for every effective medical treatment, for everyone in the covered population, entirely. Unfortunately in a world where budgets are limited, such generous coverage is possible only for the very wealthiest global citizens. Most existing healthcare systems, in both rich and poor countries, and everywhere in between, embody compromises-in terms of who is covered, what is covered, or what share of the cost is covered.

Recently, many national and global actors have fallen in line behind the Universal health coverage (UHC) banner of "UHC," a succinct label for the idea that everyone should have access to affordable and effective healthcare (Chalkidou et al., 2016; WHO, 2010). But what "affordable" means is highly situationally dependent. Moreover, even though universality takes the question of who is to be covered out of the picture, it gives little guidance as to how the balance should be struck between what is covered and what share of the cost is covered. Clearly when budgets are finite, if health services are provided free at the point of delivery, less can be provided than if the patients are required to contribute a share of the costs (Smith, 2013). 
This tradeoff is recognized by Disease Control Priorities 3, the recent project of the World Bank and the Bill and Melinda Gates Foundation, which sought to provide analysis of healthcare "best buys" for low- and middle-income countries (LMICs; Jamison and DCP3, 2018). The analysts working in this project have advocated a new form of costeffectiveness analysis, so-called "Extended Cost-Effectiveness Analysis" (ECEA; Megiddo et al., 2016; Megiddo et al., 2014; Megiddo, Klein, \& Laxminarayan, 2018; Morton \& Lauer, 2017; Nandi et al., 2016; Verguet, Kim, \& Jamison, 2016; Verguet, Laxminarayan, \& Jamison, 2015; Verguet et al., 2013). One of the defining features of ECEA is that financial protection is modeled explicitly alongside health benefits and expenditure. However, while ECEA clearly enriches the information base, decision makers may find it more taxing to move from the tables of numbers produced as part of a ECEA analysis to actual decisions about what to fund (For comparison, it is relatively easy to "read off" the action implications of a cost/quality adjusted life years [QALYs] ranking).

In this study, we offer a technical solution which can help decision makers make tradeoffs between health and financial protection. The sort of setting we have in mind is the determination of a "health benefits package"-an explicit list of all of the treatments to which a population is entitled, and at what copayment rates. The analytic and political challenges of determining a health benefits package are clearly an order of magnitude greater than those facing rich country Health Technology Assessment agencies such as the NICE or the Scottish Medicines Consortium making yes/ no decisions about particular technologies. However, for LMICs which have the ambition to rapidly scale up their healthcare provision in the face of a seriously constrained resource base, setting explicit benefits packages may be attractive for several reasons (Glassman, Giedion, \& Smith, 2017).

The tool which we propose in this study is based on multiobjective optimization. Mathematical optimization methods have been used by many as a conceptual framework for understanding health systems (Morton, 2014; Morton, Arulselvan, \& Thomas, 2018; Morton, Thomas, \& Smith, 2016b; van Baal, Meltzer, \& Brouwer, 2016; Smith \& Yip, 2016; van Baal, Morton, \& Severns, 2018). The recent reports of the ISPOR Taskforce on Constrained Optimization point the way forward to using these methods more widely in operational analysis to support the development of more efficient, more patient-centered care (Crown et al., 2018; Crown et al., 2017).

Multiobjective optimization differs from traditional optimization in that it seeks to generate not a single "best" solution, but a range of solutions which may be best depending on where the decision maker sets the tradeoff between competing objectives (Ehrgott, 2005). This can be particularly useful in a portfolio choice setting such as the determination of a benefits package, where making choices "locally" about particular technologies using different criteria may result in global choices which are not logically consistent (Morton, Keisler, \& Salo, 2016a; Salo, Keisler, \& Morton, 2011).

To illustrate how multiobjective optimization would outperform a traditional approach, let us consider an example with four health care treatments that belong to two categories. Each treatment is evaluated with respect to its health benefit and financial exposure levels. In category 1, there are treatments $\mathrm{A}$ and $\mathrm{B}$, and in category 2, $\mathrm{C}$ and $\mathrm{D}$. One item has to be chosen from each category. The decision maker for category 1 subtracts the financial exposure score from the health benefits; the decision maker for category 2 subtracts twice the financial exposure score, resulting in the overall scores seen in Table 1.

It is clear from the scores that one would choose A from Category 1 and $\mathrm{C}$ from Category 2. Let us take a look at the possible portfolios:

As seen in Table 2 and Figure 1, portfolio (A,C) is dominated by portfolio (B,D) as the latter leads to more health and less financial exposure. Multiobjective optimization is useful since one never chooses a dominated solution in the portfolio space because of local decisions guided by inconsistent values.

This example demonstrates the complexity of the decision problem when multiple technologies are considered simultaneously. In such problems, one can use 2-dimensional ECEA graphs to visualize the policy makers problem. However, it would still be challenging to evaluate all possible choice combinations (project portfolios), as the number of such portfolios would be prohibitively large. In real life settings with higher number of technologies to choose from, the complexity is even more pronounced. Multiobjective optimization can be used to support decision-making in such problems. Health benefits package design problem is a typical example where the policy makers have to compare many interventions simultaneously rather than focusing on one specific intervention. Indeed, any setting where decision makers have to make choices about multiple technologies simultaneously is a potential application for multiobjective optimization. Further applications involve territorial resource allocation problem, in which a health authority with fixed funds prioritizes various health-related investments; and the specialty-level clinical planning, in which multiple improvement techniques (or multiple specialties) are prioritized so as to improve the healthcare system with respect to multiple criteria such as patient access and efficiency. 
T A B L E 1 Overall scores

\begin{tabular}{lccc} 
Treatment & Health & Financial exposure & Overall score \\
\hline A & 105 & 55 & 50 \\
\hline B & 50 & 5 & 45 \\
\hline C & 30 & 10 & 10 \\
\hline D & 115 & 55 & 5 \\
\hline
\end{tabular}

T A B L E 2 Portfolios

\begin{tabular}{lcc} 
Portfolio & Health & Financial exposure \\
\hline $\mathrm{A}+C$ & 135 & 65 \\
$\mathrm{~A}+D$ & 215 & 110 \\
$\mathrm{~B}+\mathrm{C}$ & 80 & 15 \\
$\mathrm{~B}+\mathrm{D}$ & 165 & 60 \\
\hline
\end{tabular}

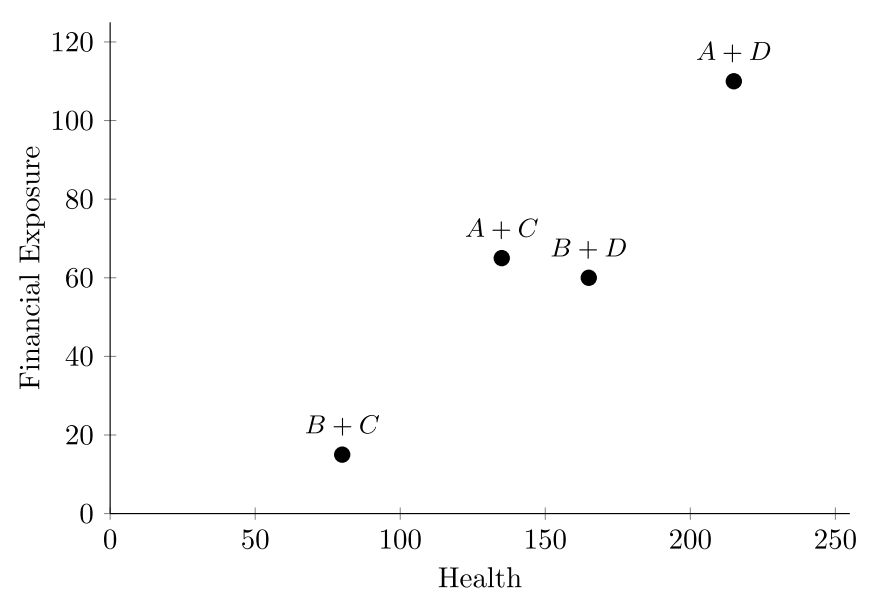

F I G U R E 1 Bicriteria analysis on portfolios

To our knowledge, the only study in the health economics literature which has previously used multiobjective optimization is [Morton, 2014], which uses the method to explore equity tradeoffs between different population subgroups who are differently disadvantaged. Morton's study uses both different model formulations and different solution algorithms from the present work.

In the following sections, we will first discuss ways to support decision makers to choose their most preferred package given the tradeoff curve that shows how different options (health benefits packages) perform with respect to the two criteria of total health benefit and financial protection. We will then focus on the problem of finding this tradeoff curve, through a biobjective optimization framework.

\section{2 | INTERACTIVE METHODS}

In this section, we suggest two approaches which may help a decision maker, who has to choose from a given set of solutions that are good to various degrees with respect to the two criteria of total health benefit and financial protection, narrow down which solution is the appropriate in the light of her objectives. Each such solution is a feasible portfolio of healthcare treatments (in the sense that the total cost of the treatments does not exceed the budget) and is also a Pareto solution, that is, there is no other solution that improves it with respect to both criteria or with respect to one criterion without sacrificing from the other.

Our first approach is as follows. If the decision maker is prepared to assume an overall objective function of the form $w H+(1-w) F P$ (where $H$ and FP denote the total health benefit and financial protection levels of a solution respectively), the situation becomes much simpler-even if she cannot specify the weight $w$. This is because the optimal 
F I G U R E 2 Tradeoff analysis [Colour figure can be viewed at wileyonlinelibrary. com]

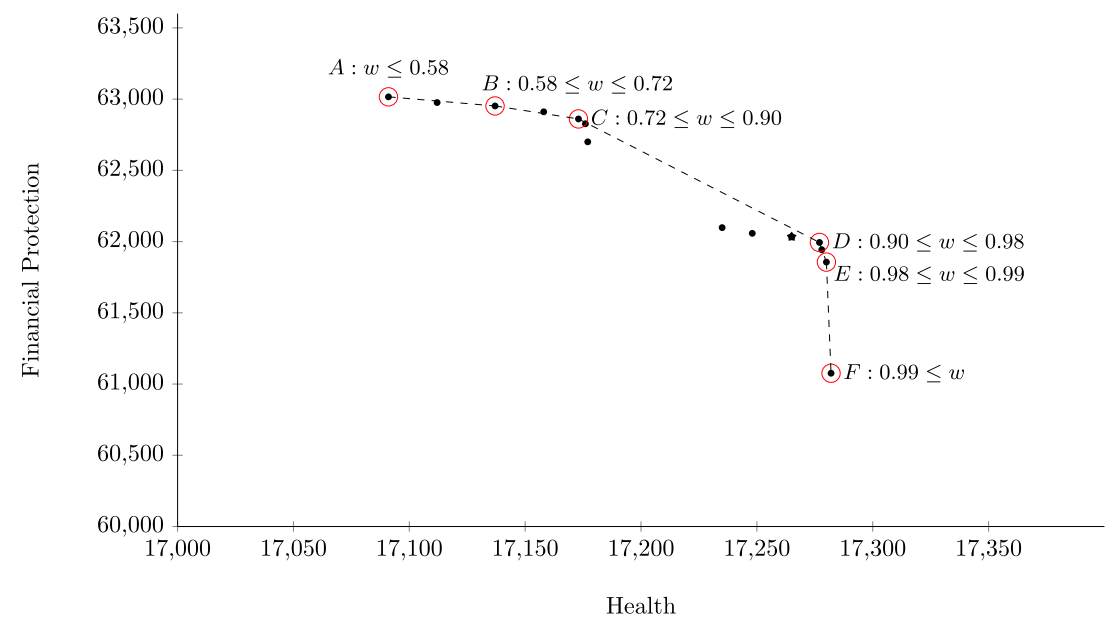

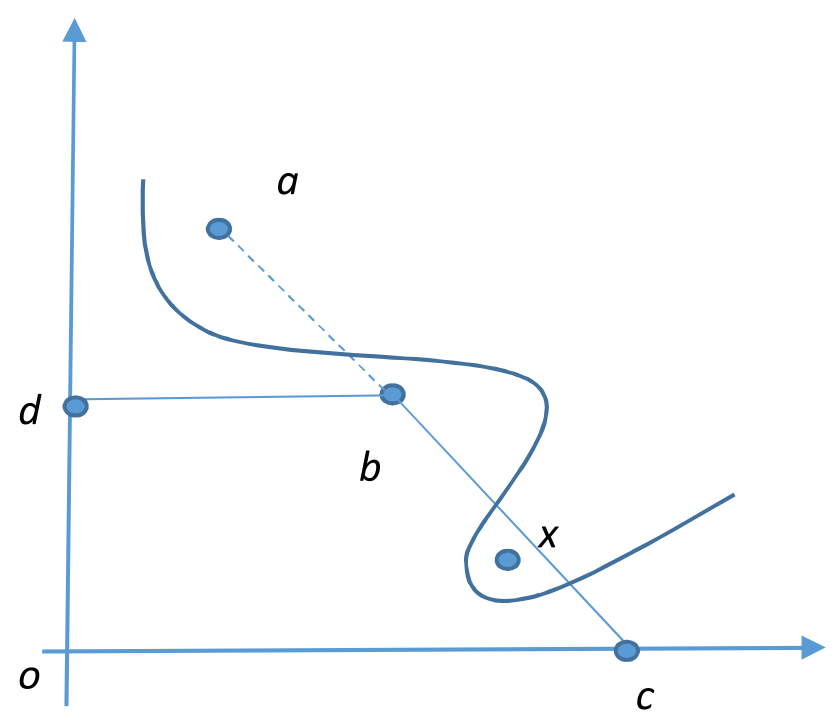

F I G U R E 3 An example depicting the contradiction to quasiconcavity [Colour figure can be viewed at wileyonlinelibrary.com]

solution must lie on the convex hull or envelope of the Pareto set. See Figure 2 for an example with 14 options. ${ }^{1}$ Note that uncertainty in the weight does not necessarily translate into uncertainty in the decision: for example, whether the weight 0.75 or 0.85 , the optimal choice is nevertheless option $C$.

Our second approach uses the following idea, the history of which is discussed in Karsu, 2013. Assume that the decision maker's objective function is $f(H, F P)$ where $f$ is some quasiconcave and increasing function, and suppose the decision maker has told us that she prefers $a$ to $b$ (see Figure 3). Then all points in the area $b c o d$ must be worse for the decision maker than $b$. To see why, suppose that there was a point $x$ contained in $b c o d$ which is superior to $b$. Then there would have to be an isoquant of $f$ which separates both $a$ and $x$ from $b$. As is evident from the Figure such an isoquant could not be convex, which contradicts the assumed quasiconcavity of $f$.

We do not provide full technical details of this idea which can be found elsewhere in greater generality than in the example studied in the current study (Korhonen, Wallenius, \& Zionts, 1984; Karsu, 2013). From our point of view, the important insight is that simple pairwise comparisons can eliminate whole regions of the Pareto set without the need to assume additivity of the decision maker's objective function. To get a sense of how this might work consider Figure 4. In the first step of this procedure, the decision maker is faced with a choice between $C$ and $D$, and choosing $D$ eliminates several points to the northwest of $C$. In the second step, the decision maker chooses between $D$ and $E$ and her choice of $D$ eliminates a further point to the southeast of $E$. The remaining five panels show how the remaining options are eliminated until a single circled point remains. The point of this example is to show that even if the Pareto set contains $N$ points, it may not be necessary to explicitly examine each of those points to come to a decision. 


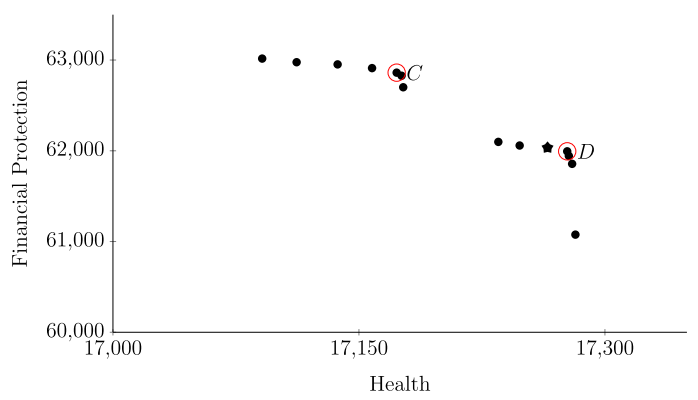

(a) Step 1: Choice

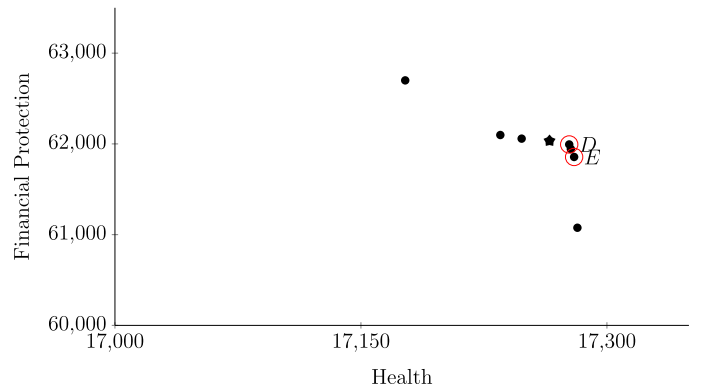

(c) Step 2: Choice

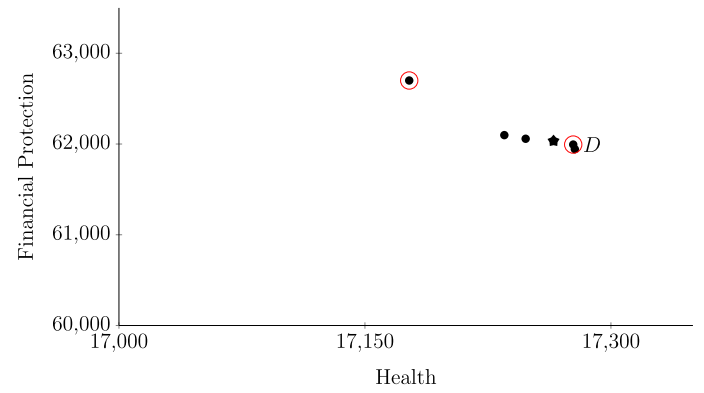

(e) Step 3

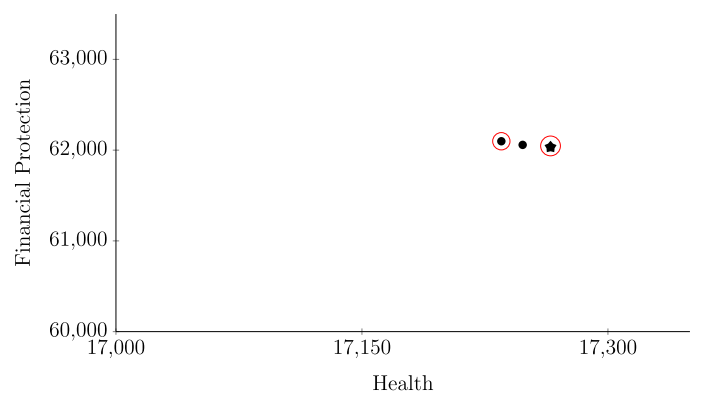

(g) Step 5

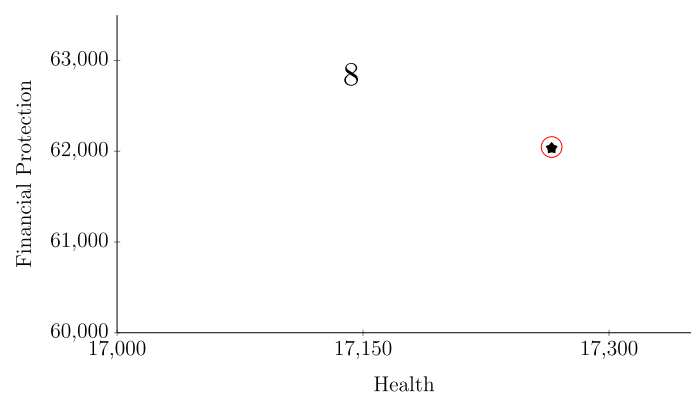

(i) Result of the interactive algorithm

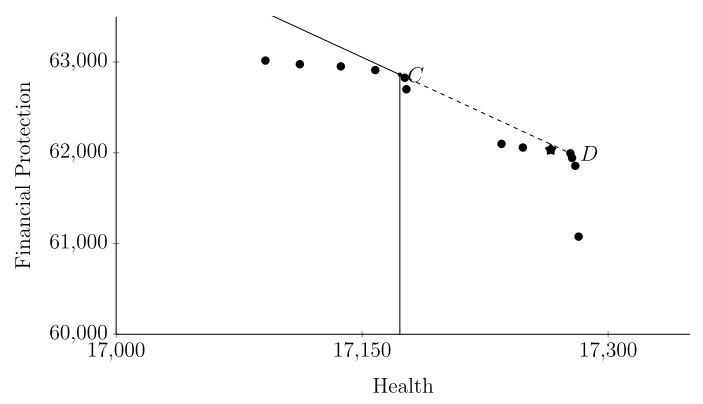

(b) Step 1: Elimination

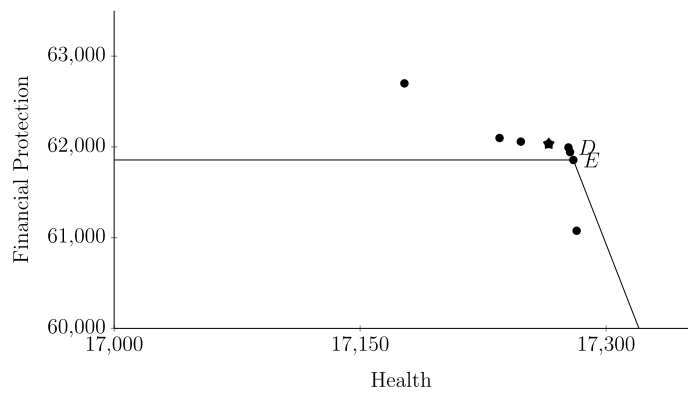

(d) Step 2: Elimination

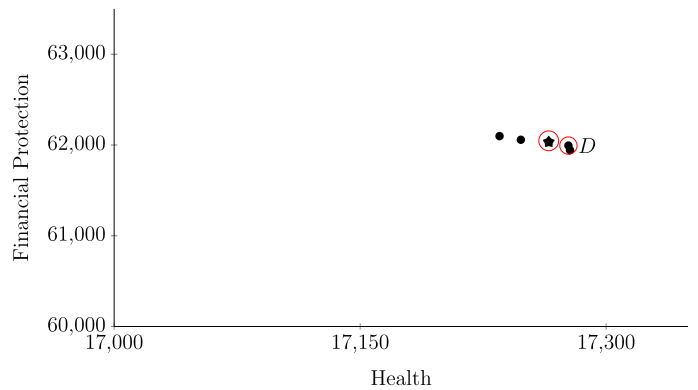

(f) Step 4

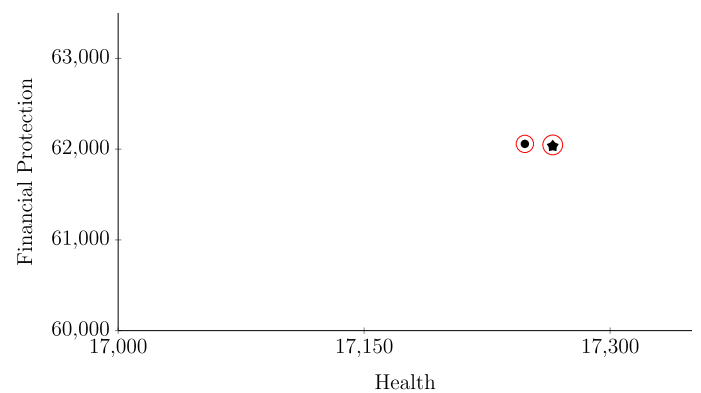

(h) Step 6

F I G U R E 4 Interactive algorithm steps [Colour figure can be viewed at wileyonlinelibrary.com] 
A relevant design question is how to choose the pairwise questions to ask the decision maker to make the most out of her answers and determine her preferred option with the least number of questions possible. A good-rule-of thumb for choosing pairs for comparison would be checking the potential of the answer in reducing the number of options. As can be seen graphically form the figures, when choosing the pairs to ask, it is better to start with solutions that lie toward the center of the frontier as this would maximize the minimum number of alternatives that can be eliminated, that is, it increases the potential to eliminate more alternatives. For example, in the first iteration, pair (C, D) is chosen because the minimum number of eliminations is the highest for this pair. If $\mathrm{C}$ is preferred, then 4 options will be eliminated while if $D$ is preferred, 5 options will be eliminated. At least 4 alternatives are eliminated whatever the choice of the decision maker is.

Note that this rule prioritizes the solutions that lie on the convex hull of the set of (not-yet-eliminated) solutions. Moreover, it tends to choose closer solutions as they will reveal more information on the tradeoff between the two criteria.

Another question arises here on how to obtain the tradeoff curve, that is the alternative health benefits packages that are Pareto solutions. For this purpose, we propose using biobjective optimization models with the objectives of maximizing total health gain and minimizing financial exposure (or equivalently, maximizing financial protection). We discuss two such mathematical models that differ with respect to how financial exposure is quantified.

\section{3 | PROPOSED MODELS}

Consider a setting where there are $n$ treatments. Each treatment $i$ is expected to generate a benefit value of $b_{i}$ per person (measured in appropriate units) and consumes $c_{i}$ units of resource per person. Let $n_{i}$ be the total number of cases requiring treatment $i$. The total amount of budget available is denoted by $B$, which typically is not sufficient to cover all treatments to their full extent.

We assume that a central decision authority decides on the copayment rates, hence covering a portion $\left(p_{i}\right)$ of the costs and leaving the rest of the treatment costs to the patients.

We consider two concerns at the same time: The concern of maximizing the total benefit achieved by the package (measured in appropriate units such as total QALYs gained or disability-adjusted life years [DALYS] averted) and minimizing financial exposure. One can use different functions to assess financial exposure (see [Verguet et al., 2016]). In this study, we maximize cases of catastrophic financial burden averted (i.e., equivalent to minimizing the number of cases with catastrophic financial burden). Any copayment that exceeds a certain threshold $(T)$ will be considered catastrophic. We will then discuss another approach based on minimizing a convex penalty function.

Let $p_{i}$ be the percentage of cost that will be covered by the package (i.e., 1-copayment rate) for treatment $i$. We will call $p_{i}$ the coverage rate. Thus, if the coverage rate is $0.7(70 \%)$, for an intervention costing $\$ 15, \$ 10.50(=\$ 15 \times 0.7)$ will be paid by the government, and the patients will have to pay the balance of $\$ 4.50$ out of their own pocket. We assume that the decision maker chooses from $m$ coverage rates as follows: $0 \quad\left(P_{0}\right) \leq P_{1} \leq P_{2} \leq \ldots \leq P_{m-1}$. Let $M=\{1,2, \ldots, m-1\}$. Let $a_{i k}, k \in M$ be a binary variable as follows:

$$
a_{i k}= \begin{cases}1, & \text { if } p_{i}=P_{k} \\ 0, & \text { otherwise }\end{cases}
$$

Note that if all $a_{i k}$ variables take a value of 0 for a treatment $i$, then the coverage rate is 0 for that treatment, which implies that $P_{0}$ is chosen.

Let $y_{i}$ be a binary variable as follows:

$$
y_{i}= \begin{cases}0, & \text { if treatment } i \text { leads to catastrophic financial burden } \\ 1, & \text { otherwise }\end{cases}
$$

$M$ is a sufficiently large number. The biobjective programming model we consider first is as follows. We call it the base model (B): 


$$
\begin{gathered}
\text { Model } B \\
\operatorname{Max} \sum_{i \in I} b_{i} n_{i} p_{i} \\
\operatorname{Max} \sum_{i \in I} n_{i} y_{i} \\
\text { s.t. } \sum_{i \in I} c_{i} n_{i} p_{i} \leq B \\
p_{i}=\sum_{k \in M} a_{i k} P_{k} \quad \forall i \in I \\
\sum_{k \in M} a_{i k} \leq 1 \quad \forall i \in I \\
\left(1-p_{i}\right) c_{i}-T \leq M\left(1-y_{i}\right) \quad \forall i \in I \\
a_{i k} \text { binary } \forall i \in I, \forall k \in M \\
y_{i} \text { binary } \forall i \in I
\end{gathered}
$$

Constraint 3 is the budget constraint. Constraint sets 2, 3, and 5 ensure that at most one coverage level is chosen for any treatment $i$. If none of the positive coverage rates $\left(P_{1}-P_{m-1}\right)$ is chosen, then no expenses are covered for that treatment, that is the copayment rate will be 1 . For each treatment $i$, the model checks whether the patients are protected from catastrophic financial burden in constraint set 4 . If the copayment $\left(\left(1-p_{i}\right) c_{i}\right)$ exceeds the threshold $T$, the left hand-side of the constraint becomes positive, forcing $y_{i}$ take a value of 0 , meaning that the patients could not be protected from catastrophic financial burden. Otherwise the $y_{i}$ variable will take a value of 1 .

The two objective functions capture conflicting intuitions about how to prioritize health expenditures. The first objective function expresses the idea that the decision maker should seek to maximize the health gains which are attributable to public funding (hence the health benefits of the different treatments are scaled by $p_{i}$ ). The second objective function captures the spirit of UHC: everyone should get the care that they need, but the cost burden on individuals should be managed so that as few people as possible are exposed to catastrophic financial burden.

One point needs to be clarified. In empirical work, the normal practice is to track catastrophic costs which are actually incurred for healthcare. One of the limitations of such measurements is that it misses cases where people have the capacity to benefit from healthcare but do not have the means to purchase it, because of limited ability to pay. The interpretation of catastrophic financial burden in our second objective function is expenditure which is required because of some medical need. Whether this cost can or cannot be met is a secondary question: what is important is that there is disutility either because of untreated illness or because of financial distress.

It is possible to extend the model in a relatively straightforward fashion to the situation where one person can bear the cost of multiple diseases, and to the situation where there are multiple population quintiles. Rather than interrupt the flow of the narrative, we place these models in Appendix A.

As mentioned before, financial exposure could be assessed using various function forms. In the above models, we have maximized the number of patients protected from financial catastrophe. The following model takes an alternative approach and minimizes a financial exposure function $f($.$) that is a convex function of the deviation from a threshold T$. This allows us to go beyond simply designating a financial burden as being catastrophic or not and to incorporate different assumptions about "how catastrophic."

$$
\operatorname{Max} \sum_{i \in I} b_{i} n_{i} p_{i}
$$

$$
\operatorname{Min} \sum_{i} n_{i} f\left(\operatorname{Dev}_{i}^{+}\right)
$$


s.t. Constraints $1,2,3,5$,

$$
\begin{gathered}
\left(1-p_{i}\right) c_{i}=T+\operatorname{Dev}_{i}^{+}-\operatorname{Dev}_{i}^{-} \forall i \in I \\
\operatorname{Dev}_{i}^{+} \geq 0 \quad \forall i \in I \\
\operatorname{Dev}_{i}^{-} \geq 0 \quad \forall i \in I
\end{gathered}
$$

Constraint set 7 is used to determine the difference between expenditures required for treatment $i\left(\left(1-p_{i}\right) c_{i}\right)$ and the threshold T. Positive and negative deviations are denoted as $\operatorname{Dev}_{i}{ }^{+}$and $\operatorname{Dev}_{i}{ }^{+}$, respectively.

The above model is not necessarily linear due to the use of function $f($.). To obtain a linear programming model, one can use piecewise linear convex functions that approximate (nonlinear) convex financial exposure functions as follows.

We first divide the range for possible values of $\mathrm{Dev}_{i}{ }^{+}$into intervals defined by levels. Let $L_{1}, \ldots, L_{i n t}$ be the deviation levels (see Figure 5 where int $=5$ ). In each interval between two consecutive levels, we assume linearity. Let $w_{1}, \ldots, w_{i n t}$ be the slopes of the corresponding intervals as seen in Figure 5. Since the function is convex, we have $w_{1}<w_{2}<\ldots<w_{\text {int }}$. Given a set of levels and the corresponding slopes for the intervals, one can find $f(x)$ for any deviation value $x$ by detecting the interval $x$ belongs to. For example, suppose that $x$ belongs to interval 2 (i.e., $L_{1}<x<L_{2}$ ), then $f(x)=w_{1} L_{1}+w_{2}\left(x-L_{1}\right)$. In the mathematical model, we will define new decision variables that show the amount any deviation $x$ spans in each interval ( Dev $_{i k}$ variables used below).

The resulting linear programming model is as follows. We call it the convex financial exposure function model (C).

\section{Model $C$}

$$
\operatorname{Max} \sum_{i \in I} b_{i} n_{i} p_{i}
$$

$$
\operatorname{Min} \sum_{i} n_{i} f_{i}
$$

s.t. Constraints $1,2,3,5,7,8,9$

$$
\begin{gathered}
\operatorname{Dev}_{i}^{+}=\sum_{k=1}^{\mathrm{int}} \operatorname{Dev}_{i k} \\
\operatorname{Dev}_{i 1} \leq L_{1} \\
L_{1} \delta_{i 2} \leq \operatorname{Dev}_{i 1} \\
\operatorname{Dev}_{i k} \leq\left(L_{k}-L_{k-1}\right) \delta_{i k} \forall k=2, \ldots, \text { int } \\
\left(L_{k}-L_{k-1}\right) \delta_{i k+1} \leq \operatorname{Dev}_{i k} \forall k=2, \ldots, \text { int }-1 \\
f_{i}=\sum_{j=1}^{\text {int }} w_{j} \operatorname{Dev}_{i j} \\
\delta_{i k} \text { binary } \forall i \in I, \forall k=2, \ldots, \text { int }
\end{gathered}
$$

Constraint set 10 defines the $\operatorname{Dev}_{i k}$ values, which show the amount that $\operatorname{Dev}_{i}{ }^{+}$spans in each interval. Constraints 11-14 make sure that $\operatorname{Dev}_{i k}$ value for an interval $k$ cannot be positive unless the previous intervals are filled up first, using the binary variables $\delta_{i k} \mathrm{~s}$. That is, for example if $\operatorname{Dev}_{i}{ }^{+}$is in interval $2\left(L_{1}<\operatorname{Dev}_{i}{ }^{+}<L_{2}\right)$, then $\operatorname{Dev}_{i 1}=L_{1}$ and $\operatorname{Dev}_{i 2}=\operatorname{Dev}_{i}{ }^{+}-L_{1} \cdot \delta_{i 2}=1$ due to constraint 11 and the rest of the $\delta_{i k}$ variables will have a value of 0 . Note that there is no $\delta_{i 1}$ defined.

$f_{i}$ variables keep the per-person financial exposure level for treatments (i.e., $f_{i}=f\left(\operatorname{Dev}_{i}^{+}\right)$) and are calculated using the $\operatorname{Dev}_{i k}$ variables and the corresponding interval slopes as explained above. 


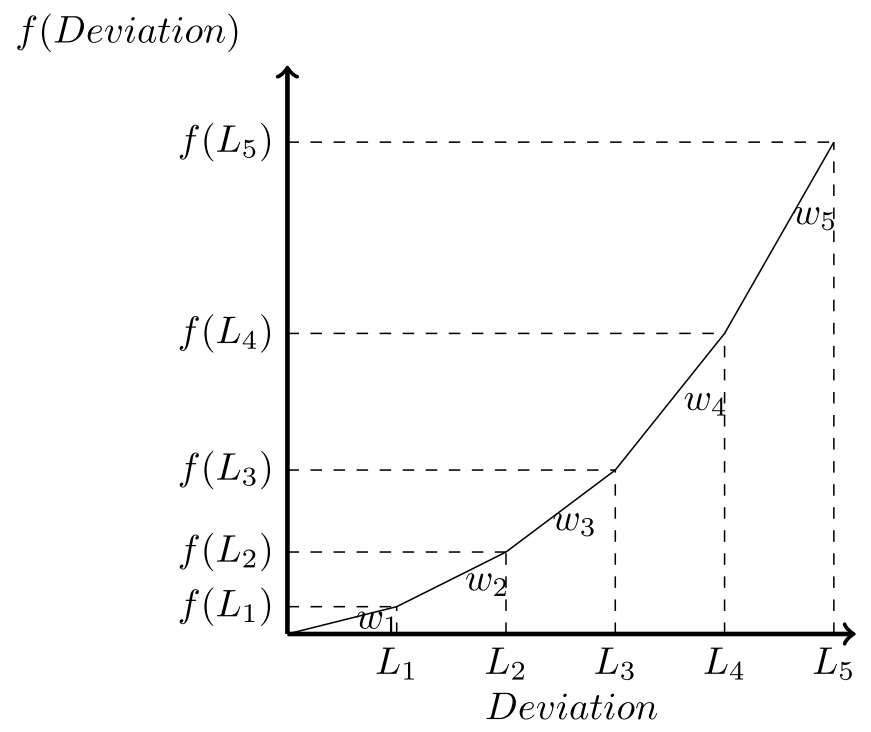

F I G U R E 5 A Piecewise linear convex function with five intervals

\section{4 | NUMERICAL ILLUSTRATION}

We illustrate our model with data relating to the Malawian health benefits package as described in (Ochalek et al., 2018). The data table reproduced in Appendix B shows the list of treatments considered in this study (treatments that have very small number of cases or that have negative net DALYs averted are excluded).

To solve the biobjective model, we use the epsilon constraint approach (Ehrgott, 2005) using a stepsize of 1 for the total health benefit (since the number of cases values are scaled, this corresponds to an actual stepsize of 1000), to find the Pareto solutions. The approach is explained in Appendix C. The algorithm is coded in MATLAB and all mathematical models are solved in CPLEX 12.7. The algorithm is run on a personal computer with i5-4590 $3.30 \mathrm{GHz}$ processor and 8 GB RAM. Computation times are calculated in central processing unit (CPU) seconds.

We set $T=5$ and considered five coverage levels $(M=\{1,2,3,4\})$, which are $0,0.25\left(P_{1}\right), 0.4\left(P_{2}\right)$, and $0.9\left(P_{3}\right)$ and $1\left(P_{4}\right)$ and report results for two different cardinalities of $M$. For the cases with three coverage levels $(M=\{1,2\})$, we used $0,0.4\left(P_{1}\right)$ and $0.9\left(P_{2}\right)^{2}$

We considered three different scenarios for the total budget. Note that the total cost covering all treatments to the full extent would be around $\$ 264.5$ million. If all of them were covered to $90 \%$ that would require around $\$ 238$ million (M). We tried three levels for the budget parameter: $70 \mathrm{M}$ (around $30 \%$ of the total cost of covering all treatments to the highest coverage level), $90 \mathrm{M}(38 \%), 110 \mathrm{M}(46 \%)$.

All of the computational times were under $5 \mathrm{~s}$. The results for different budget levels (70,90, and 110M) and different number of coverage options ( 3 coverage level options and 5 coverage level options) are summarized in Figure $6 .^{3}$

One can see that the solution sets for larger budget levels dominate the solution sets for smaller budgets, which is to be expected. The budget level also affects the number of solutions obtained. In general, we expect the number of solutions to be small for extreme (very high or very low) budget levels. Indeed, when the budget is sufficiently large to cover all treatments in the package fully, there is only a single solution with the best levels of both objectives. When the budget is very low, the number of feasible options, hence the number of nondominated solutions becomes small. At intermediate budget levels, we expect the number of solutions to become relatively large as the tradeoff comes into play (compared to high budget levels) and the number of alternative portfolios is larger compared to low budget levels. Note that the budget levels we chose for demonstration are all intermediate.

If, however, there are a small number of "large" projects and several "small" projects, which are included in some solutions while being left out at others, there may be sudden jumps at the frontier. See Appendix D for a more detailed discussion.

Figure 6 also shows the effect of the number of coverage rate options $(m)$ on the results. One can see that for a fixed budget, increasing the number of possible coverage levels leads to better solutions in terms of both objectives. This implies that having flexibility with respect to the coverage options would increase the overall benefit of a universal health care package. On the other hand, using large numbers of possible copayment levels may not be convenient from 
F I G U R E 6 Pareto solutions for different budget levels and coverage level options. Each dot represents a nondominated (Pareto) point. CFB, Catastrophic financial burden; DALYs, disability-adjusted life years

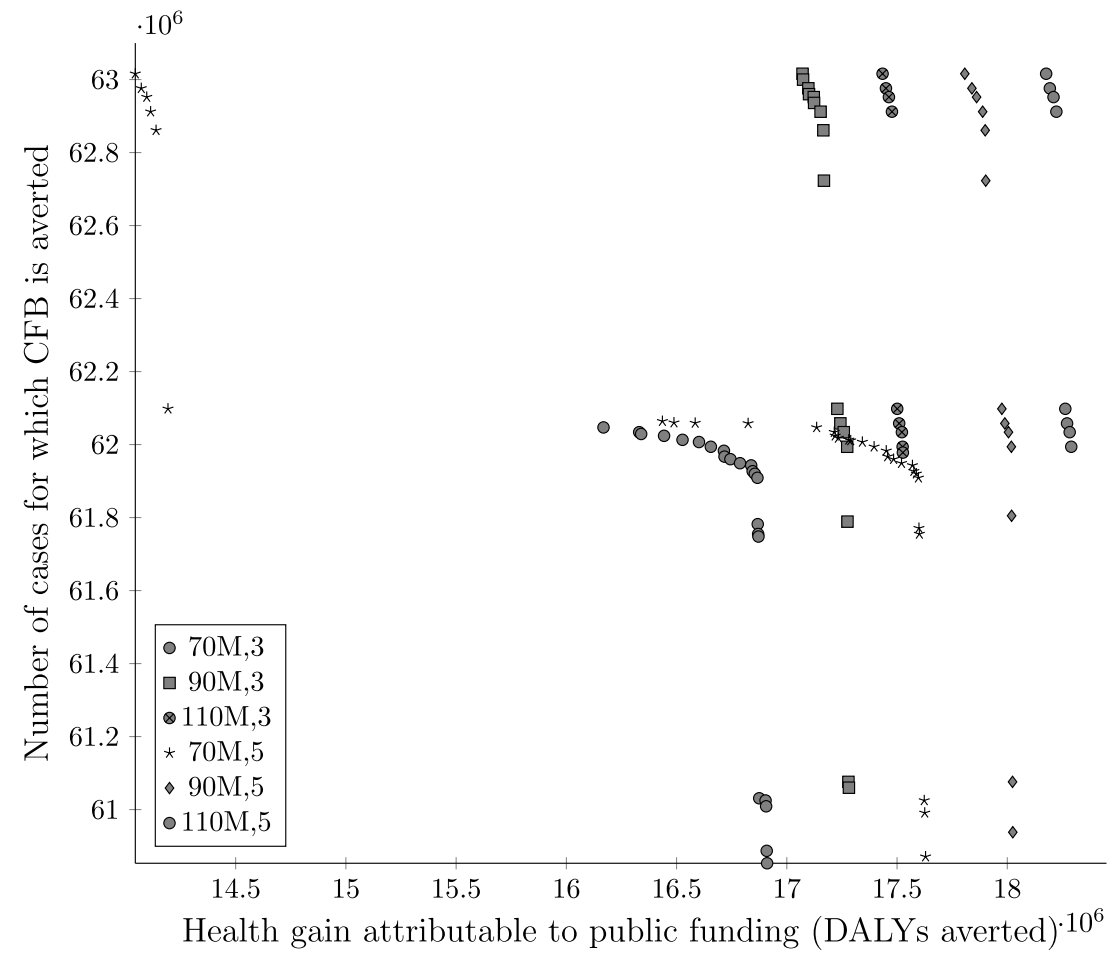

a practical point of view. This is a design decision that should be made considering pros and cons of the options. Such analyses can help the policy makers see whether increasing the number of alternative copayment levels is justified.

Detailed analysis of solutions for the setting with 5 coverage levels and $90 \mathrm{M}$ budget are seen below. Figure 7 shows the coverage levels of different treatments across the Pareto solutions. The treatments are ordered with respect to their core index (average coverage level across all Pareto solutions.) It is possible to detect the must-do treatments (2-7, 9,11-17,19-20,25-26,28,31,33-34,38-39, and 41-42) and the do not do treatments (18,23-24, and 36-37). Moreover, it is seen that treatments 1,29,35, and 46 are fund at least partially and 29,35,46,21,10,32,30, and 40 are consider funding fully.

For comparison, we redid the analysis, this time using a convex financial exposure function. We again assumed that $B=\$ 90$ million and there are five coverage levels $0\left(P_{0}\right), 0.25\left(P_{1}\right), 0.4\left(P_{2}\right), 0.9\left(P_{3}\right)$, and $1\left(P_{4}\right)$. We set $T=5$, that is, the financial exposure function is a convex function of copayment amounts above 5 .

10 levels are chosen for the piecewise linear financial exposure function, starting from 5 and increasing in increments of 5 units up to 50 (note that the maximum unit cost is 47.78). Figure 8 shows the function used and Figure 9 shows the results.

In general, we observe that the solutions obtained using the two methods are very similar. However, the second method returns more solutions as it allows alternative solutions to be obtained by changing the deviation amounts above the threshold. In the first approach, there is no distinction between copayment levels that result in costs at the same side of threshold, they all contribute to the exposure function by the number of cases. However, how much one deviates from the threshold plays an important role in the second one. For example, for treatment 43, one either uses a coverage level of 0 (in which case, the patient will have catastrophic financial burden) or 0.9 (in which case the patient will not have catastrophic financial burden) in the first method: 0.25 and 0.4 levels are not sufficient to save the individuals from catastrophic expenses. However, when the second method (convex penalty function) is used, such levels also matter since they lead to different amounts of deviation from the threshold. We obtain alternatives where $40 \%$ of the expenses are covered for treatment 43 when the second exposure function is used. Similar observations can be made for another high-unit-cost treatment, treatment 45.

To further the case that models B and C are closely equivalent, we ran both models for a number of different problem instances, obtained the ranks of treatments with respect to their core index and calculated the correlation between the rank orders obtained in both models. For this purpose, we generated different scenarios with $25,50,75$, and 100 treatments. For each level of $n$, we created 5 problem instances, in which $n_{i}, c_{i}$, and $b_{i}$ are randomly generated between ranges $(2,16,000),(1,50)$, and $(1,60$; These ranges are similar to the ranges in Malawi data). We set the budget 

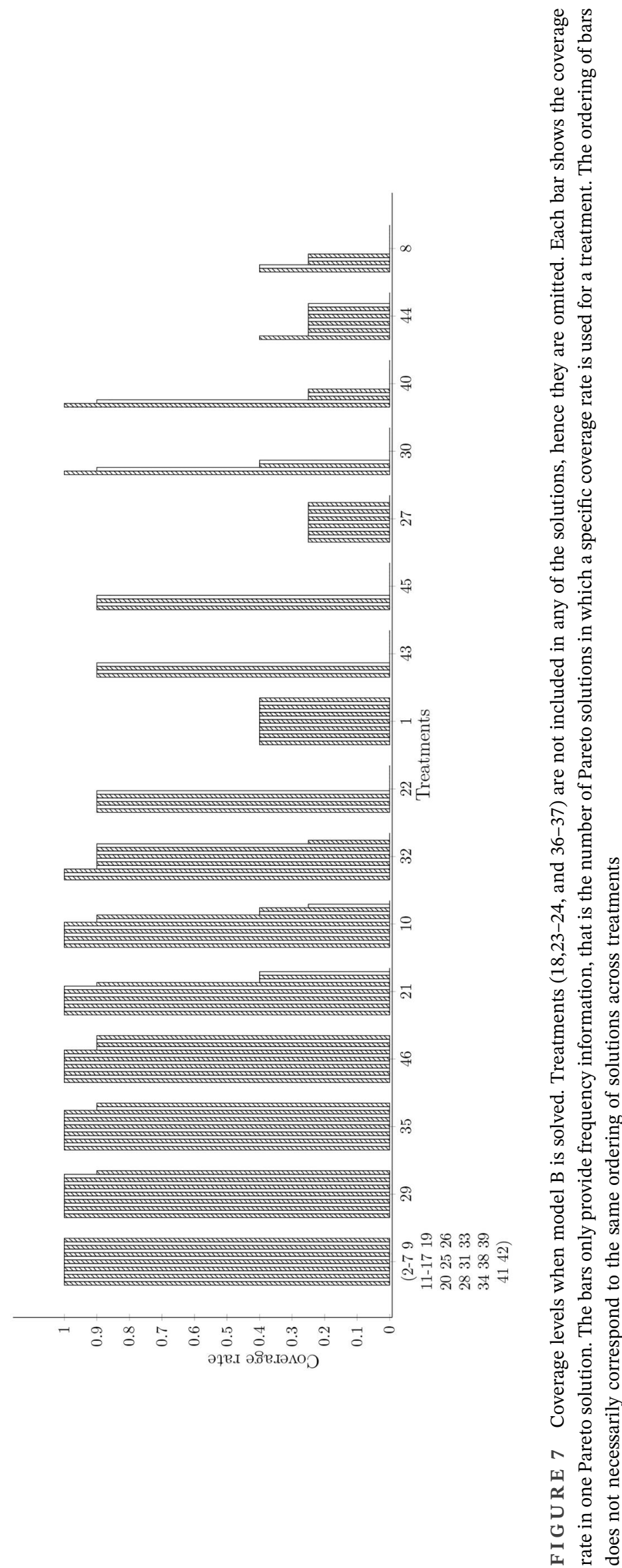
F I G U R E 8 Financial exposure function used in the analysis

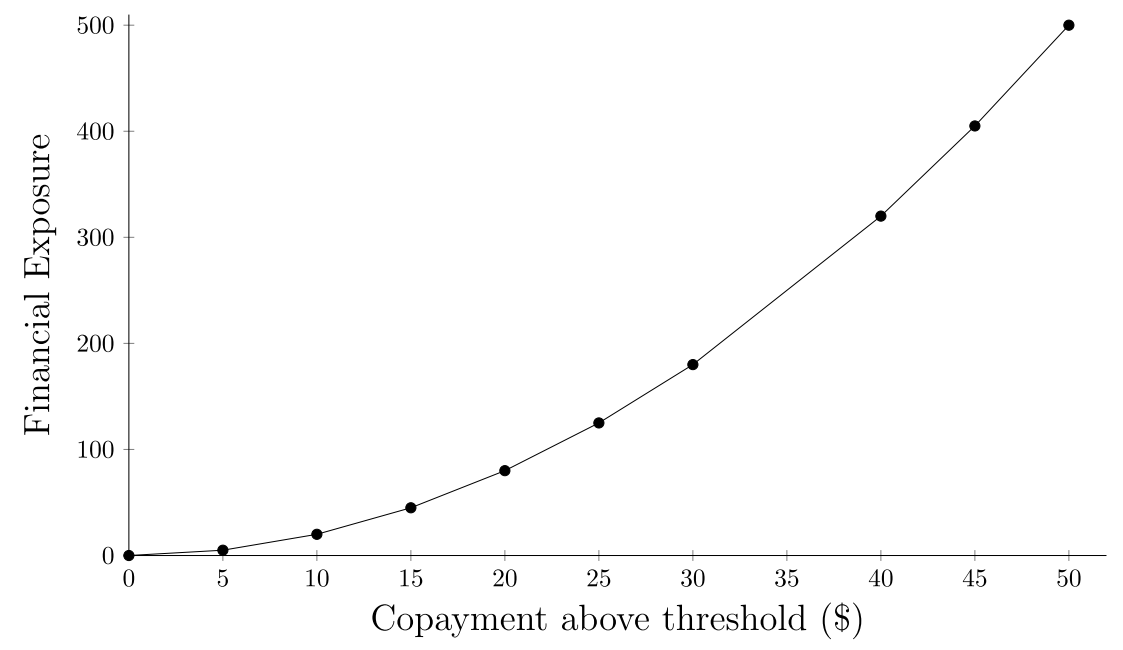

as $50 \%$ of the total money that would be required if all treatments are funded fully and set $T=25$. The average and minimum correlation values for different sized instances are seen in Table 3. The results indicate that the solutions are not sensitive to the metric used to measure financial exposure.

The solution times were negligible for smaller instances (under five seconds for $n=25$ and under five minutes for $n=50$, using a stepsize of 1.). As the problem size increases the solution time increases for both models but more so in model C. This is because model C typically has more Pareto solutions than model B and each single objective model solved to obtain these solutions is larger with more decision variables and constraints compared to the ones in model $\mathrm{B}$. In larger instances, we increased the stepsize for model $\mathrm{C}$ to obtain the Pareto solutions in reasonable time. For $n=75$, we increased the stepsize to 1000 for model $\mathrm{C}$ and the average solution times for were 1000 and $3500 \mathrm{~s}$ for models B and C, respectively. For $n=100$, we increased the stepsize to 5000 for model $\mathrm{C}$ and the average solution times were 2640 and $2765 \mathrm{~s}$ for models B and C, respectively.

\section{5 | CONCLUSION}

The purpose of this study has been to study how decision makers might tradeoff health and financial protection benefits in the context of the construction of a health benefits package for a country which is moving to UHC. Our reason for using a multiobjective optimization frame to study this problem is that making an explicit tradeoff between health and financial protection may be extremely difficult. Nevertheless, analysis can still provide assistance in helping decision makers avoid health benefits packages which are internally inconsistent. Our study is a first step in the analysis of this question and allows plenty of scope for additional work.

Below, we discuss a number of extensions that could be handled within the multiobjective optimization framework by modifying the mathematical models that we use.

Ministries of Health typically have as an objective the maximization of population health when prioritizing the funds which are under the control of the Ministry of Health. We interpret this as meaning that the Ministry wishes to maximize health benefits attributable to these funds and define the total health benefit objective accordingly. All outof-pocket payments are accounted irrespective of whether they are below or above the threshold, when calculating the total benefit of the healthcare package attributable to public funding. Out-of-pocket payments below the threshold do not increase the second objective, which is related to the financial burden, as this objective focuses only on the catastrophic costs. It is, however, still possible to accommodate different views on how total health benefit or financial burden should be calculated by modifying the mathematical models and changing the forms of the objective functions. For example, the Ministry of Health may choose only to count the health benefits when they are entirely paid for public funds and no copayment is required; or the financial protection function could be a more smooth nonlinear function of the cost burden on patients. Hence, the proposed biobjective optimization framework would still be relevant and useful.

Note that there may be interdependencies between treatments, making the costs, benefits or demand of treatments depend on the decision on other treatments. In such cases, the mathematical programming formulation could be modified to include these sorts of interactions, as long as they could be measured (the interested reader is referred to 

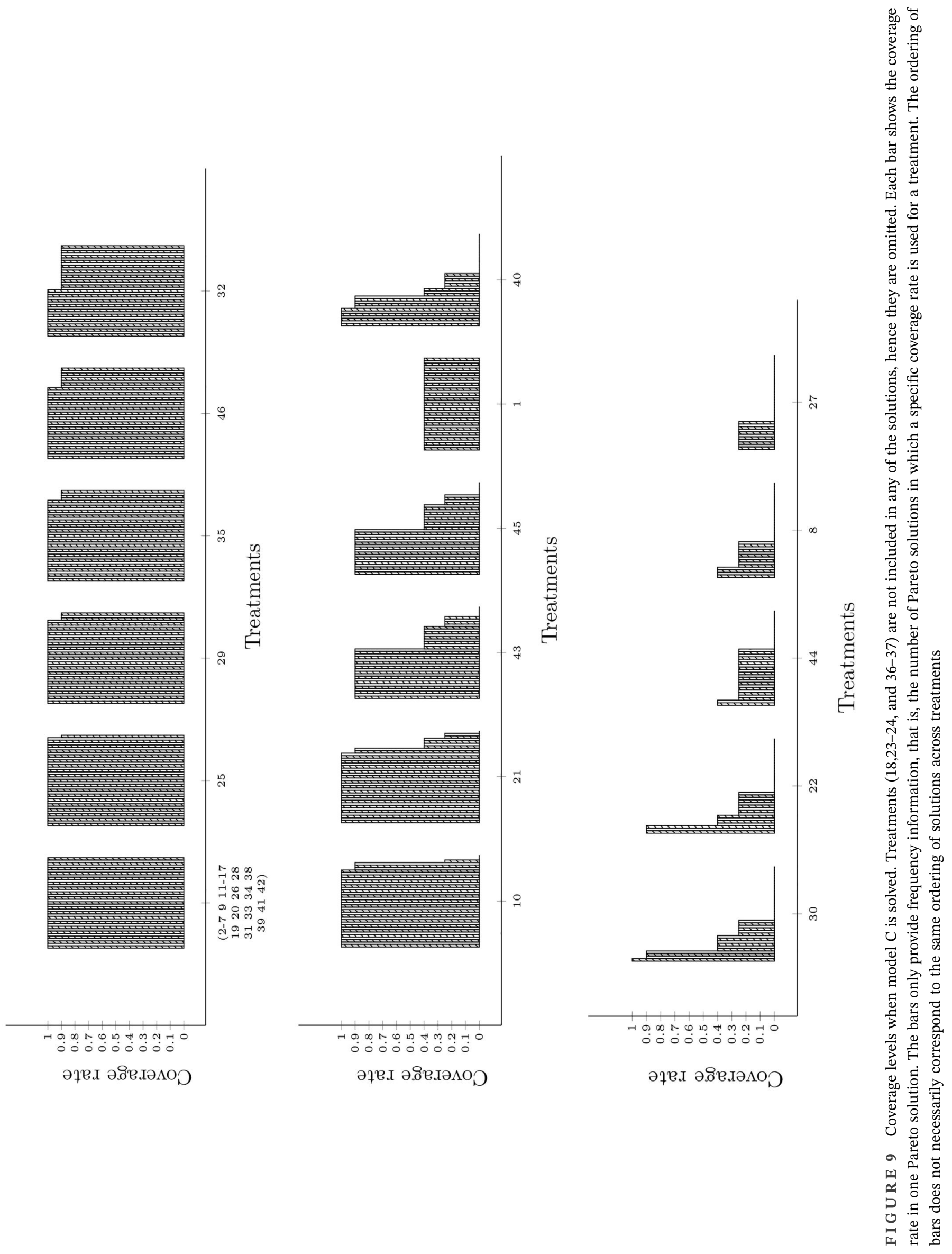


\begin{tabular}{lll}
68 & WI LEY - & $\begin{array}{c}\text { Health } \\
\text { Economics }\end{array}$ \\
\cline { 3 - 3 }$n$ & Avg. correlation & \\
\hline 25 & 0.894 & Min. correlation \\
\hline 50 & 0.958 & 0.829 \\
75 & 0.952 & 0.945 \\
100 & 0.961 & 0.921 \\
\hline
\end{tabular}

[Winston, 2004] for more discussion on mathematical modeling). Such interdependencies between interventions are addressed in the paper by (Hauck et al., 2019), where different ways of modeling these interdependencies and incorporating them into an optimization framework are proposed. In the health benefit package design problem that we consider, as long as measurement can be made, it is possible to incorporate these interactions into the optimization setting in similar ways to those discussed by (Hauck et al., 2019).

Further research could be performed in this domain to address various related questions. For example, it would be important to know how robust our models are to a range of approaches to modeling the financial protection benefits. Also, benefits may differ according to what delivery platforms and human resources are available (Hauck et al., 2019; Morton et al., 2016b; van Baal et al., 2018). Furthermore, in a development setting, there may not be a single decision maker as a significant share of the funding may come from international donors (Morton et al., 2018). Lastly, establishing a benefits package in an environment where there is copayments involves making tradeoffs between different population subgroups with different levels of ability to pay (Morton, 2014). Equity concerns for patient groups with different abilities to pay can be incorporated into the biobjective optimization framework by defining catastrophic expenses in relation to ability to pay, that is defining population group based thresholds, as discussed in Appendix A. Such a model would decide whether a person faces catastrophic burden based on the socioeconomic level of that person. Maximizing the number of people saved from such expenses would implicitly prioritize the ones with low thresholds. Compared to the base approach that does not take the ability to pay into account when defining catastrophic expenses, such an extension may change recommendations (the set of Pareto solutions) in favor of lower income quintiles, however, we expect the main observations made on the tradeoff between total health benefit and total financial protection to still hold. We leave the integration of these complexities into our models as a challenge for further research.

\section{ACKNOWLEDGMENT}

The authors are grateful to Dr Itamar Megiddo for his feedback on a previous version of the manuscript.

\section{CONFLICT OF INTEREST}

The authors have declared that they have no conflict of interest.

\section{ORCID}

Özlem Karsu (D) https://orcid.org/0000-0002-9926-2021

Alec Morton (1) https://orcid.org/0000-0003-3803-8517

\section{ENDNOTES}

${ }^{1}$ This is the Pareto set of the example health benefits package design problem formulated with Malawian case study data with 4 coverage levels and a $90 \mathrm{M}$ budget, which will be explained in the following sections.

2 These levels are set only for demonstration purposes. The framework can be used with any number of coverage levels.

3 To ensure readability, we do not show one extreme solution of $70 \mathrm{M}, 3$ scenario which had $7.44 \times 10^{6}$ DALYs averted and $63.016 \times 10^{6}$ cases for which CFB is averted.

\section{REFERENCES}

Chalkidou, K., Glassman, A., Marten, R., Vega, J., Teerawattananon, Y., Tritasavit, N., ... Culyer, A. J. (2016). Priority-setting for achieving universal health coverage. Bulletin of the World Health Organization, 94(6), 462-467.

Crown, W., Buyukkaramikli, N., Sir, M. Y., Thokala, P., Morton, A., Marshall, D. A., ... Pasupathy, K. S. (2018). Application of constrained optimization methods in health services research: Report 2 of the ispor optimization methods emerging good practices task force. Value in Health, 21(9), 1019-1028. 
Crown, W., Buyukkaramikli, N., Thokala, P., Morton, A., Sir, M. Y., Marshall, D. A., ... Pasupathy, K. S. (2017). Constrained optimization methods in health services research-an introduction: Report 1 of the ispor optimization methods emerging good practices task force. Value in Health: The Journal of the International Society for Pharmacoeconomics and Outcomes Research, 20(3), 310-319.

Ehrgott, M. (2005). Multicriteria optimisation. Berlin, Germany: Springer.

Glassman, A., Giedion, U., \& Smith, P. C. (2017). What's in, what's out: Designing benefits for universal health coverage. Washington, DC: Center for Global Development.

Hauck, K., Morton, A., Chalkidou, K., Chi, Y. L., Culyer, A., Levin, C., ... Smith, P. C. (2019). How can we evaluate the cost-effectiveness of health system strengthening? A typology and illustrations. Social Science \& Medicine, 220, 141-149.

Jamison, D. T., \& DCP3. (2018). Universal health coverage and intersectoral action for health: Key messages from disease control priorities, 3rd edition. Lancet, 391, 1108-1120.

Karsu, O. (2013). Using holistic multicriteria assessments: The convex cones approach. New York, NY: Wiley.

Korhonen, P., Wallenius, J., \& Zionts, S. (1984). Solving the discrete multiple criteria problem using convex cones. Management Science, 30(11), 1336-1345.

Megiddo, I., Colson, A., Chisholm, D., Dua, T., Nandi, A., \& Laxminarayan, R. (2016). Health and economic benefits of public financing of epilepsy treatment in India: An agent-based simulation model. Epilepsia, 57(3), 464-474.

Megiddo, I., Colson, A. R., Nandi, A., Chatterjee, S., Prinja, S., Khera, A., \& Laxminarayan, R. (2014). Analysis of the universal immunization programme and introduction of a rotavirus vaccine in India with indiasim. Vaccine, 32(Suppl 1), A151-A161.

Megiddo, I., Klein, E., \& Laxminarayan, R. (2018). Potential impact of introducing the pneumococcal conjugate vaccine into national immunisation programmes: An economic-epidemiological analysis using data from India. BMJ global health, 3(3), e000636.

Morton, A. (2014). Aversion to health inequalities in healthcare prioritisation: A multicriteria optimisation perspective. Journal of Health Economics, 36, 164-173.

Morton, A., Arulselvan, A., \& Thomas, R. (2018). Allocation rules for global donors. Journal of Health Economics, 58, 67-75.

Morton, A., Keisler, J., \& Salo, A. (2016a). Multicriteria portfolio decision analysis for project selection. New York, NY: Springer.

Morton, A., \& Lauer, J. A. (2017). Comparing apples and oranges: Strategies to weigh health against other social values. Washington, DC: Center for Global Development.

Morton, A., Thomas, R., \& Smith, P. C. (2016b). Decision rules for allocation of finances to health systems strengthening. Journal of Health Economics, 49, 97-108.

Nandi, A., Colson, A. R., Verma, A., Megiddo, I., Ashok, A., \& Laxminarayan, R. (2016). Health and economic benefits of scaling up a homebased neonatal care package in rural India: A modelling analysis. Health Policy and Planning, 31(5), 634-644.

Ochalek, J., Revill, P., Manthalu, G., McGuire, F., Nkhoma, D., Rollinger, A., ... Claxton, K. (2018). Supporting the development of a health benefits package in Malawi. BMJ global health, 3(2), 1-14.

Salo, A., Keisler, J., \& Morton, A. (2011). Portfolio decision analysis: Methods for improved resource allocation. New York, NY: Springer.

Smith, P. C. (2013). Incorporating financial protection into decision rules for publicly financed healthcare treatments. Health Economics, 22, 180-193.

Smith, P. C., \& Yip, W. N. (2016). The economics of health system design. Oxford Review of Economic Policy, 32(1), 21-40.

van Baal, P., Meltzer, D., \& Brouwer, W. (2016). Future costs, fixed health care budgets and the decision rules of cost effectiveness analysis. Health Economics, 25(2), 237-248.

van Baal, P., Morton, A., \& Severns, J. L. (2018). Health care input constraints and cost effectiveness analysis decision rules. Social Science \& Medicine. 200, 59-64.

Verguet, S., Kim, J. J., \& Jamison, D. T. (2016). Extended cost-effectiveness analysis for health policy assessment: A tutorial. PharmacoEconomics, 34(9), 913-923.

Verguet, S., Laxminarayan, R., \& Jamison, D. T. (2015). Universal public finance of tuberculosis treatment in India: An extended cost-effectiveness analysis. Health Economics, 24(3), 318-332.

Verguet, S., Murphy, S., Anderson, B., Johansson, K. A., Glass, R., \& Rheingans, R. (2013). Public finance of rotavirus vaccination in India and Ethiopia: An extended cost-effectiveness analysis. Vaccine, 31(42), 4902-4910.

WHO. (2010). World health report 2010: Financing for universal health coverage. Geneva, Switzerland: World Health Organization.

Winston, W. L. (2004). Operations research: Applications and algorithms, 4th ed. Thomson/Brooks/Cole.

\section{SUPPORTING INFORMATION}

Additional supporting information may be found online in the Supporting Information section at the end of this article.

How to cite this article: Karsu Özlem, Morton A. Trading off health and financial protection benefits with multiobjective optimization. Health Economics. 2021;30:55-69. https://doi.org/10.1002/hec.4176 